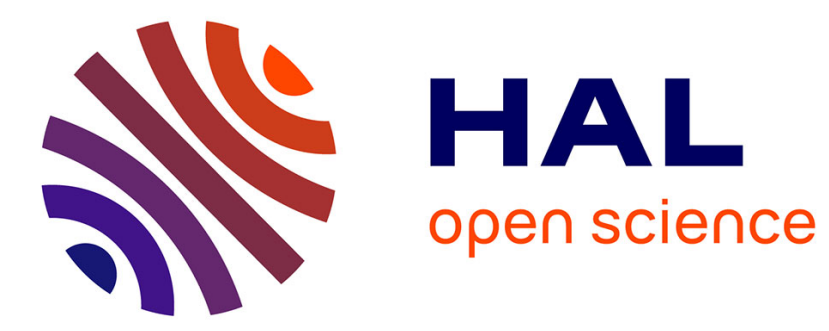

\title{
Les aventures de la cour : courses, fêtes et divertissements
}

Marine Roussillon

\section{To cite this version:}

Marine Roussillon. Les aventures de la cour : courses, fêtes et divertissements. Littératures classiques, 2019, N¹00 (3), pp.171. 10.3917/licla1.100.0171 . hal-03006902

\section{HAL Id: hal-03006902 \\ https://hal.science/hal-03006902}

Submitted on 16 Nov 2020

HAL is a multi-disciplinary open access archive for the deposit and dissemination of scientific research documents, whether they are published or not. The documents may come from teaching and research institutions in France or abroad, or from public or private research centers.
L'archive ouverte pluridisciplinaire HAL, est destinée au dépôt et à la diffusion de documents scientifiques de niveau recherche, publiés ou non, émanant des établissements d'enseignement et de recherche français ou étrangers, des laboratoires publics ou privés. 


\section{Roussillon Marine, "Les aventures de la cour : courses, fêtes et divertissements ", Littératures classiques, 2019/3 ( $\left.N^{\circ} 100\right)$, p. 171-183.}

\section{Les aventures de la cour : courses, fêtes et divertissements}

« Enfin, le Roi, [...] avec les trois Aventuriers qui l'accompagnaient, alla, le Sabre à la main, abattre les Têtes de quatre Hydres ${ }^{1}$ ». La Gazette du 26 février 1667 dresse ainsi un portrait du jeune Louis XIV en aventurier, au carrefour de la chevalerie et de la mythologie. La phrase est extraite du récit d'une course de têtes organisée pour le carnaval : les hydres sont fictives et leurs têtes sont de simples ballots que les cavaliers doivent abattre pour montrer leur virtuosité. L'aventure est un jeu, à la fois exercice militaire - il s'agit pour les courtisans de s'exercer à la guerre en temps de paix - et spectacle divertissant. Le récit qui en est fait, dans un périodique dont l'objet principal est l'actualité internationale, conjugue lui aussi revendication de puissance guerrière et recherche du plaisir du lecteur. L'aventure et son récit se situent ainsi d'emblée à la frontière entre pratique guerrière et divertissement, entre nouvelles et récits fictionnels.

Les courses données pour le carnaval de 1667 ne sont pas un événement isolé. Dans les années 1660, les divertissements de la cour mobilisent régulièrement un imaginaire de l'aventure et jouent de son équivocité pour configurer les relations entre plaisir et pouvoir. Les mots « aventure » et « aventurier » apparaissent ainsi dans plusieurs récits de la première fête organisée par Louis XIV à Versailles, Les Plaisirs de l'île enchantée, ainsi que dans la relation du Grand Carrousel du roi de 1662 publiée par l'Imprimerie royale en 1670. En produisant des interprétations de l'aventure, entre guerre et plaisir, les récits de ces divertissements définissent dans un même mouvement les valeurs de la cour et leur propre valeur d'écrit.

Désigner les divertissements comme des aventures, c'est d'abord un moyen de les associer à la pratique guerrière qui définit la noblesse et ainsi de manifester la force du roi et de ses courtisans. C'est aussi faire référence à un imaginaire romanesque et construire une représentation agréable de cette force. Les récits de fêtes proposent alors une expérience agréable du pouvoir royal. Force et plaisir, éthique guerrière et esthétique galante, peuvent cependant entrer en tension : c'est ce que nous verrons pour finir à travers l'étude de deux aventuriers, l'un bien réel - le duc de Saint-Aignan - et l'autre fictif - Sostrate, le héros des Amants magnifiques de Molière.

\footnotetext{
${ }^{1}$ La Gazette (N²6), du 26 février 1667, p. 197-199. Passage cité dans la base de données intertextuelles MOLIÈRE21, dans la rubrique « Le Ballet des Muses dans la Gazette» :

http://moliere.paris-sorbonne.fr/base.php?Le_Ballet_des_Muses_dans_la_Gazette
} 


\section{Courses et carrousels : l'aventure comme « image de la guerre »}

La Gazette du 26 février 1667 relate les divertissements organisés à Versailles pour les derniers jours du carnaval : trois jours de fêtes qui commencent par un bal masqué, se poursuivent avec une journée de courses de têtes et se terminent par un second $b^{2}{ }^{2}$. Les courses du deuxième jour développent le thème traditionnel de la rivalité entre nations : le duc de Saint-Aignan est vêtu «à la Persienne », Louis XIV porte un habit «à la Hongroise », Monsieur est habillé « à la Turque » et le duc d'Enghien « en Indien ». Le récit désigne les cavaliers qui prennent part aux courses tantôt comme des « chevaliers », tantôt comme des « aventuriers »:

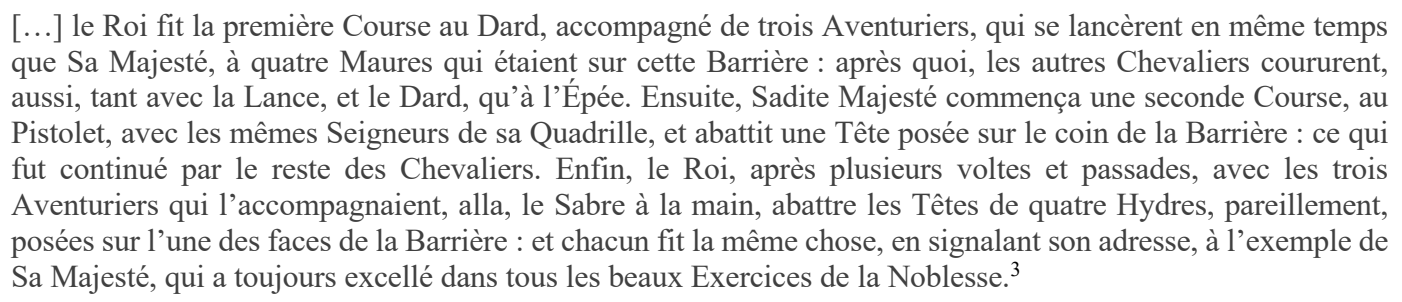

Les mêmes termes sont employés, et désignés comme synonymes, par le gazetier Robinet dans la lettre en vers qu'il consacre à ces courses :

$$
\begin{aligned}
& \text { [...] une GROSSE TROUPE, } \\
& \text { Laquelle avait le Vent en Poupe : } \\
& \text { C'était celle des CHEVALIERS, } \\
& \text { Ou, si l'on veut, AVENTURIERS. }
\end{aligned}
$$

Quelques années plus tard, en 1670, lorsque paraît enfin le récit officiel du grand carrousel du roi de 1662, les mots d' « aventurier» et de « chevalier» sont encore utilisés comme deux termes équivalents pour désigner les participants 5 .

Le rapprochement de ces deux mots tend à définir l'aventurier comme un noble et comme un guerrier. « Chevalier » est d'abord, selon Furetière, « le premier degré d'honneur de l'ancienne milice, qu'on donnait avec certaines cérémonies à ceux qui avaient fait quelque exploit signalé qui les distinguait des autres gens de guerre ». Le dictionnaire poursuit : "Ainsi on appelle Chevaliers, les gens issus de haute et ancienne Noblesse, ou qui ont été faits Chevaliers par les Princes ${ }^{6} »$. Les récits des courses associent donc l'aventure à la noblesse, définie comme ordre guerrier. Ces usages du terme « aventurier » rejoignent la première définition qu'en donne le dictionnaire de l'Académie, et qui fait de l'aventurier un homme de guerre motivé par sa seule gloire

qui cherche les aventures, les occasions de la guerre, espèce de volontaire. Il se disoit autrefois particulierement de ceux qui alloient volontairement à la guerre, sans recevoir de solde, et sans s'obliger aux gardes et aux autres fonctions militaires qui ne sont que de fatigue. ${ }^{7}$

L'aventure est ainsi liée à une pratique guerrière spécifique, tout entière tournée vers la recherche de la gloire et caractéristique de la noblesse.

Les courses racontées par la Gazette ou par la relation de 1670 apparaissent comme des substituts de cette pratique héroïque de la guerre. La Gazette les définit comme des « exercices de la noblesse » : des entraînements destinés à entretenir les compétences guerrières des nobles en temps de paix. Cette idée est amplement développée

\footnotetext{
${ }^{2}$ Sur ces courses, les dernières auxquelles Louis XIV a participé, voir S. Castelluccio, Les Carrousels en France du XVI $I^{e}$ u XVIII siècle, L'Insulaire-Les Editions de l'Amateur, 2002, p. 34-35.

${ }^{3}$ Loc. cit.

4 Robinet, Lettre en vers à Madame du 27 février 1667, sur le site MOLIÈRE 21: http://moliere.parissorbonne.fr/base.php?Robinet\%2C_Lettre_en_vers_à_Madame_du_27_février_1667

${ }^{5} \mathrm{Ch}$. Perrault, Courses de testes et de bague faites par le roy et par les princes et seigneurs de sa cour en l'année 1662, Paris, Imprimerie royale, 1670, par exemple p. 2, 7, 65, 67...

${ }^{6}$ A. Furetière, Dictionnaire universel, La Haye, A. et R. Leys, 1690, article « chevalier ».

${ }^{7}$ Dictionnaire de l'Académie Française, première édition, 1694, Article «aventurier ». Consulté en ligne : http://artflproject.uchicago.edu/content/dictionnaires-dautrefois
} 
dans le récit du grand carrousel du roi. Les courses y sont désignées comme des « images de la guerre », de « feints combats ${ }^{8}$, « utiles pour bannir l'oisiveté et la mollesse qui corrompent ordinairement tous les fruits de la paix ${ }^{9} »$.

Ce sont encore d'illustres écoles de discipline militaire, où l'on apprend mieux qu'en toutes autres le véritable métier de la guerre, puisque non seulement ils forment l'adresse du corps, mais qu'ils augmentent en quelque sorte le courage, en allumant le désir de la gloire, qu'on peut nommer l'ame de la valeur. ${ }^{10}$

La relation officielle des fêtes versaillaises de 1664, Les Plaisirs de l'île enchantée, utilise, elle aussi, le terme « aventure » pour désigner des combats feints : non plus des courses, mais un ballet dans lequel les danseurs parmi lesquels figurent le roi et bon nombre de courtisans - jouent le rôle de chevaliers combattant des monstres ${ }^{11}$. Toujours dans cette relation, le mot «aventure » désigne encore un autre type de combat : une scène de chasse dans laquelle deux princes triomphent d'un sanglier. C'est dans La Princesse d'Élide, comédie-ballet de Molière créée pendant le deuxième jour des fêtes et reproduite dans la relation :

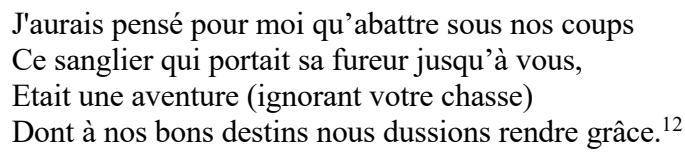

Dans le Divertissement royal de 1670, c'est à nouveau un combat contre un sanglier qui est désigné comme une « aventure merveilleuse ${ }^{13}$ ». Courses, ballet, chasse : dans ces récits de fêtes, l'aventure désigne toujours une forme stylisée, ludique et divertissante de la pratique guerrière qui caractérise la noblesse.

En désignant le roi et ses courtisans comme des aventuriers, les récits des courses publient leur force et leur bravoure : ils mobilisent une définition traditionnelle de la noblesse comme ordre militaire pour faire l'éloge du roi. Ce portrait est d'autant plus intéressant, au début du règne personnel de Louis XIV, que le roi n'a pas eu l'occasion de manifester sa vertu guerrière sur les champs de bataille. Sa puissance ne peut alors s'affirmer que dans le cadre ludique des divertissements de cour.

En 1670, lorsque paraît la relation du carrousel de 1662, les enjeux de ce portrait se sont déplacés : la monarchie est revenue à une politique de guerre, et il ne s'agit plus seulement de donner à voir la puissance du roi, mais aussi d'affirmer la légitimité de ses combats, contre tous ceux qui l'accusent d'excès d'orgueil ou de vénalité ${ }^{14}$. Le début de la relation s'y emploie, en soulignant la distance qui sépare les courses de la guerre, et en en proposant plusieurs interprétations. Les courses y sont d'abord désignées comme des « exercices» permettant à la noblesse de s'entraîner en temps de paix. Une fois représentées dans le récit, elles deviennent des exemples utiles à l'éducation du Dauphin, dédicataire de l'ouvrage. Enfin, l'insertion de ce récit dans le récit plus vaste de l'histoire du roi transforme le carrousel en épreuve, qui rend visibles les motifs cachés des actions du roi. C'est dans ces « feints combats » que se révèle la vérité de la guerre et le secret du pouvoir :

Ce n'est pas sans raison que ceux qui ont entrepris d'écrire l'Histoire des Grands Hommes, ont crû qu'il ne suffisoit pas d'apprendre à la Postérité les Batailles qu'ils avoient gagnées, et les conquestes qu'ils avaient faites, mais qu'il fallait encore lui faire connoître quels avoient été leurs plaisirs et leurs divertissemens. En effet, tous ces Exploits merveilleux, qui font l'objet de l'admiration des peuples, peuvent être des effets du vice aussi bien que de la vertu, [...] souvent une crainte servile du jugement des hommes, une soif déréglée de leurs louanges, un désir brutal de vengeance ou quelque passion plus honteuse ont produit les mêmes miracles que le véritable amour de la gloire. Il n'en est pas de même du temps qui est destiné à leurs plaisirs [...]. S'ils témoignent alors ce même amour pour la gloire, cette horreur pour l'oisiveté et cette ardente inclination au

${ }^{8}$ Ch. Perrault, Courses de testes et de bague..., op. cit., « Épistre à Monseigneur le Dauphin », n. p. et p. 1.

${ }^{9}$ Ibid., p. 2.

${ }^{10}$ Loc. cit.

${ }^{11}$ L'île enchantée, course de bagues, collation ornée de machines, comédie mêlée de danse et de musique, ballet du Palais d'Alcine; feu d'artifice : et autres fêtes galantes et magnifiques, faites par le Roi, à Versailles, le $7^{e}$ mai 1664, et continuées plusieurs autres jours, Paris, Ballard, 1664, n. p.

${ }^{12}$ Molière, La Princesse d'Elide, I, 3 (v. 267-270), dans L'île enchantée..., op. cit., p. 37 et dans Molière, CEuvres complètes, G. Forestier et C. Bourqui (dir.), Paris, Gallimard, 2010, t. I, p. 553.

${ }^{13}$ Molière, Les Amants magnifiques, V, 1, dans Molière, Euvres complètes, éd. cit., t. II, p. 989.

${ }^{14}$ Sur le développement du discours et des images attaquant Louis XIV, voir I. Boitel, L'image noire de Louis XIV : ProvincesUnies, Angleterre, 1668-1715, Ceyzérieu, Champ Vallon, 2016. 
travail, qui paraît quelquefois en eux dans les occasions où il s'agit de leur grandeur et de leur sûreté ; c'est alors certes qu'on peut assurer que la vertu est l'âme et le principe de toutes leurs actions. ${ }^{15}$

Les courses, parce qu'elles s'inscrivent dans un entre-deux entre pratique guerrière et divertissement, parce qu'elles sont une guerre « feinte », apparaissent ainsi comme une épreuve capable de révéler ce qui reste invisible dans la guerre véritable, de dévoiler les vices ou de faire éclater la vertu du souverain. Dans ce glissement de la manifestation de force à la légitimation de la violence, de l'exercice d'une puissance à la représentation d'un pouvoir, la mise en récit joue un rôle essentiel. C'est dans l'écriture que se construit la distance qui fonde l'aventure et la différencie de la guerre : dans le récit, la violence guerrière se mue en plaisir et la force du roi devient un pouvoir agréable, capable de susciter l'adhésion.

\title{
Convertir la violence en plaisir
}

La désignation des courses et carrousels comme « aventures », si elle témoigne d'une relation entre aventure et pratiques guerrières, tend aussi à rapprocher les courses d'un autre univers, celui de la fiction et particulièrement du roman. La lettre que le gazetier Robinet consacre aux festivités de février 1667 souligne ce lien en désignant les courses comme « un CARROUSEL des plus charmants, / Tel qu'on en voit dans les ROMANS » ${ }^{16}$. L'usage des majuscules associe les courses non plus à la pratique guerrière mais à la fiction romanesque. La rime, quant à elle, fonde cette association sur le plaisir suscité par l'une et par l'autre : c'est le «charme » des courses qui les apparente au roman. Le récit des courses, en reconduisant ce plaisir, devient alors lui-même une forme de roman d'aventures. Le plaisir de la cour, divertie par le carrousel, est relayé et redoublé par le plaisir du lecteur - ainsi lui-même agrégé à l'élite de la cour. Guerre feinte, l'aventure est donc aussi une guerre plaisante, agréable à voir et surtout à lire. Le récit prend le pas sur la pratique des courses, et la distance qui sépare la guerre et l'aventure devient le lieu d'une élaboration esthétique visant le plaisir.

Cette proximité avec le roman est particulièrement sensible dans la relation des Plaisirs de l'île enchantée. Le ballet désigné comme aventure est en effet inspiré d'un roman de chevalerie, le Roland furieux de l'Arioste, qui a donné lieu à plusieurs réécritures aussi bien romanesques que dramatiques dans la première moitié du siècle ${ }^{17}$. L' «aventure » du ballet d'Alcine est bien une aventure de roman: celle des chevaliers prisonniers de la magicienne, puis libérés par les enchantements de Mélisse et les combats de Roger. Dans ces récits, l'aventure est assimilée à un divertissement. La relation manuscrite offerte à certains courtisans s'achève ainsi :

Lors un coup de tonnerre, suivi de plusieurs éclairs, marque la destruction du palais, qui est aussitôt réduit en cendres par un feu d'artifice, qui finit cette sixième entrée et met fin à cette aventure, et aux divertissements de l'Ile enchantée. ${ }^{18}$

Dans le changement de point de vue, du ballet à son récit, des courtisans aux spectateurs et aux lecteurs, le sens et la valeur de l'aventure sont redéfinis : l'exercice devient divertissement, le combat devient objet de plaisir. La relation des fêtes rédigée par Marigny met en lumière cette conversion de la violence en plaisir :

\begin{abstract}
Alcine parut comme une désespérée, et lors un grand coup de Tonnerre suivi d'une infinité d'éclairs marqua la ruine de son Palais qui fut embrasé par un feu d'artifice. Jamais l'on n'a vu d'incendie plus agréable ; l'air, la terre, et l'eau étaient couverts tantôt de fusées volantes, et tantôt de gerbes de feu, tantôt mille serpenteaux s'élançaient de l'Île sur les spectateurs, et il y en eut tel qui tombant parmi les Dames fut assez indiscret, pour se glisser, et crever en des endroits forts sujets au feu. Voilà quelle fut la fin de l'aventure, et des plaisirs de l'Île enchantée d'Alcine. ${ }^{19}$
\end{abstract}

L'aventure est à nouveau désignée comme l'un des « plaisirs » offert par la fête. L'incendie et la ruine, qui figurent ici la violence des combats, deviennent, dans le spectacle et dans son récit, " agréables ». Le plaisir des spectateurs et des lecteurs est de deux ordres. D'une part, le feu d'artifice suscite l'admiration par sa magnificence ; d'autre part, l'« indiscrétion » des « serpenteaux » de feu est l'occasion d'une allusion à la sexualité des spectatrices qui

\footnotetext{
${ }^{15}$ C. Perrault, Courses de testes et de bague..., op. cit., p. 1.

${ }^{16}$ Robinet, Lettre en vers à Madame du 27 février 1667, loc. cit.

${ }^{17}$ Entre autres, Jean Mairet, Le Roland furieux, tragicomédie, Paris, A. Courbé, 1640 ; la deuxième entrée du Ballet royal de la nuit (Paris, Ballard, 1653) ; Gabriel Gilbert, Les Amours d'Angélique et de Médor, tragédie, Paris, T. Quinet, G. de Luynes, Mauger, 1664. Voir Cioranescu, L'Arioste en France des origines à la fin du XVIII siècle, Paris, Presses modernes, 1938.

18 Bizincourt, Les plaisirs de l'isle enchantée, ordonnéz par Louis XIV, roy de France et de Navarre, à Versailles, le 6 may 1664, BNF, Ms Fr 7834, fo72r.

${ }^{19}$ Marigny, Relation des divertissements que le roi a donné aux reines dans le parc de Versailles, écrite à un gentilhomme qui est présentement hors de France, Paris, C. Barbin, 1664, p. 51-52.
} 
suscite un plaisir grivois. L'aventure se charge alors de valeurs multiples : à la fois violence guerrière et spectacle agréable, objet d'une admiration partagée pour le pouvoir du roi et d'un voyeurisme licencieux.

La relation officielle des fêtes met elle aussi en scène l'équivocité du plaisir et l'ambivalence de l'aventure. À l'aventure hérö̈que des chevaliers, font écho les « douces aventures » de la magicienne Alcine. Après avoir vu en songe la ruine de son palais, celle-ci déclare :

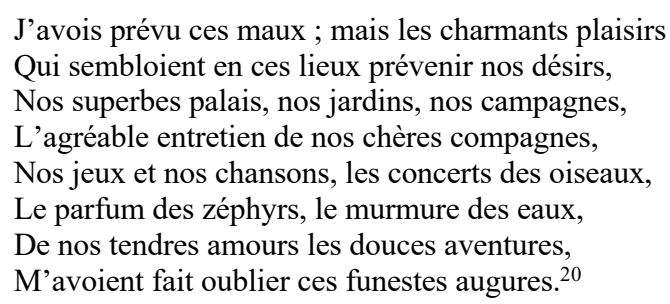

Les «aventures» d'Alcine, elles aussi désignées comme des sources de plaisir parmi d'autres («l'agréable entretien », les « jeux »), sont des aventures érotiques. Loin de mettre en lumière la puissance de la magicienne, elles mettent en danger son royaume : elles lui font oublier toute prudence et la conduisent à sa perte. Du singulier au pluriel, du masculin au féminin, l'aventure change de valeur : l'aventure du roi signale sa force et fonde son pouvoir; les aventures de la magicienne sont autant de débauches qui réduisent son pouvoir à néant. Les mettre ainsi en parallèle dans le récit permet de distinguer le pouvoir du roi de celui de la magicienne ${ }^{21}$. Là où Louis donne du plaisir à sa cour, et au-delà à tous les lecteurs de la relation des fêtes, en convertissant sa force en spectacle agréable, Alcine utilise sa puissance pour son propre plaisir, et place spectateurs et lecteurs en position de voyeurs.

La mise en scène et en récit de l'aventure participe ainsi de la construction du portrait du roi : il s'agit de donner à voir un pouvoir à la fois fort et agréable, de faire de la violence du pouvoir un objet de plaisir et d'adhésion. Cette conversion est réalisée par une esthétique galante (dont l'objet principal est le plaisir) ${ }^{22}$, fondée sur la surprise et le merveilleux, et qui apparente le récit de fête au roman, à la tragi-comédie, au poème héroïque ou, plus tard, à l'opéra. Le mouvement des relations de fêtes est celui d'un cheminement dans le domaine royal, décrit comme un espace enchanté. Au fil de ce parcours, les courtisans et le lecteur sont arrêtés par des apparitions surprenantes. Ainsi, le récit de la troisième journée des Plaisirs de l'île enchantée décrit l'arrivée de la cour devant un «grand rond d'eau » :
On fit paroître sur ce rond d'eau, dont l'étendue et la forme sont extraordinaires, un rocher situé au milieu d'une île couverte de divers animaux, comme s'ils eussent voulu en défendre l'entrée.
Deux autres îles plus longues, mais d'une moindre largeur, paroissoient aux deux côtés de la première ; et toutes trois, aussi bien que les bords du rond d'eau, étoient si fort éclairées, que ces lumières faisoient naitre un nouveau jour dans l'obscurité de la nuit.
Leurs Majestés étant arrivées n'eurent pas plutôt pris leur place, que l'une des deux îles qui paroissoient aux côtés de la première, fut toute couverte de violons fort bien vêtus. L'autre, qui lui étoit opposée, le fut en même temps de trompettes et de timbaliers, dont les habits n'étoient pas moins riches.
Mais ce qui surprit davantage, fut de voir sortir Alcine de derrière le rocher, portée par un monstre marin d'une grandeur prodigieuse. ${ }^{23}$

Les apparitions s'enchaînent, de plus en plus surprenantes : le rocher entouré d'animaux, les musiciens, et enfin, « ce qui surprit d'avantage », le monstre marin. Les tournures impersonnelles ( « on fit paraître », « fut couverte ») et les notations de temps («pas plus tôt », « en même temps ») contribuent à l'effet de surprise en occultant tous les préparatifs. La temporalité est bien celle de l'aventure. L'île, les musiciens, le monstre adviennent: ils surgissent dans l'espace enchanté sans préparation. Le récit souligne le caractère surnaturel de ces apparitions : le rond d'eau est «extraordinaire », la lumière contre-nature ( « un nouveau jour dans l'obscurité de la nuit»), le monstre «prodigieux ». La relation des divertissements versaillais de 1668, rédigée par Félibien, adopte une esthétique similaire. Le récit suit le parcours des courtisans dans le domaine de Versailles :

\footnotetext{
${ }^{20}$ L'île enchantée, ..., op. cit., n. p. ; dans Molière, Euvres complètes, éd. cit., t. I, p. 590.

${ }^{21}$ Voir mon article, « Amour chevaleresque, amour galant et discours politique de l'amour dans Les Plaisirs de l'île enchantée (1664)», K. Dickhaut et A. Viala (dir.), Littératures classiques, 69, automne 2009, « Les Discours artistiques de l'amour à l'âge classique », p. 65-78.

${ }^{22}$ Sur l'esthétique galante, voir A. Viala, La France galante, Paris, Puf, 2008.

${ }^{23}$ L'île enchantée, ..., op. cit., n. p. ; dans Molière, Euvres complètes, éd. cit., t. I, p. 589.
} 
Leurs Majestez [...] arrivèrent, après plusieurs détours, dans un cabinet de verdure, et de figure pentagone, où aboutissent cinq allées. Au milieu de ce Cabinet il y a une fontaine dont le bassin est bordé de gazon. De ce bassin sortoient cinq tables en manière de bufets, chargées de toutes les choses qui peuvent composer une collation magnifique [...]; et à bien considérer la beauté de ce lieu, l'on auroit pu dire autrefois que les hommes n'auroient point eû de part à un si bel arrangement, mais que quelques Divinitez de ces bois auroient employé leurs soins pour l'embellir de la sorte ${ }^{24}$.

Le festin, comme l'île d'Alcine, est décrit comme une apparition. L'effacement de la durée et des acteurs de sa préparation permet de le présenter comme l'effet d'une action quasi-divine : l'action du roi. Dans les relations officielles des fêtes versaillaises de 1664, 1668 et 1674, le roi est désigné comme celui qui fait advenir l'aventure, dans un geste dont la puissance semble surnaturelle ${ }^{25}$. L'esthétique de l'aventure, fondée sur la surprise et le merveilleux, contribue à figurer le caractère absolu du pouvoir royal et à en faire un objet de plaisir. La lecture du récit des divertissements de la cour se mue en expérience agréable du pouvoir sans limite du roi.

En s'appropriant une esthétique fondée sur la surprise et le merveilleux, les relations des fêtes se font donc récits d'aventures et convertissent la violence du pouvoir royal en plaisir de lecture. À la fois aventurier et ordonnateur de l'aventure, le roi y réalise la synthèse d'une éthique noble fondée sur la vertu guerrière et l'amour de la gloire et d'une esthétique galante fondée sur la surprise.

\title{
Ambivalences de l'aventure : Saint-Aignan et Sostrate
}

Dans les récits de fêtes, l'aventure est ainsi ambivalente. D'une part, elle est associée à la violence guerrière : elle est l'occasion de manifester la force, la bravoure et l'amour de la gloire qui caractérisent la noblesse, définie comme ordre militaire. D'autre part, elle relève d'un imaginaire fictionnel et d'une esthétique romanesque caractérisée par le merveilleux. Tenir ensemble ces deux aspects de l'aventure permet aux fêtes et à leurs récits d'opérer la conversion de la violence du pouvoir en plaisir. Cependant, cette ambivalence n'est pas sans poser problème. Un pouvoir qui emprunte ainsi ses formes à la fiction court le risque d'apparaître lui-même comme fictif, illusoire ou mensonger: l'esthétique de l'aventure peut être retournée contre le pouvoir qui se l'est appropriée et le désigner comme une imposture.

L'un des « aventuriers » des courses de 1667 a ainsi été qualifié de « Dom Quichotte », la référence à l'univers romanesque venant cette fois discréditer la revendication de force et de bravoure. Il s'agit du duc de Saint-Aignan, un courtisan proche du roi, premier gentilhomme de sa chambre et à ce titre principal organisateur de la fête des Plaisirs de l'île enchantée, élevé au rang de duc et pair au début de l'année 1664 en raison de sa fidélité au roi pendant la Fronde et de ses hauts faits militaires. Si Saint-Aignan a pu prouver sa valeur sur les champs de bataille, il semble aussi s'être plu à la publier en s'appropriant un imaginaire romanesque. En 1663, il fait enregistrer au parlement de Paris la grâce que lui accorde le roi pour avoir tué trois hommes qui l'avaient attaqué. La Gazette rend compte de l'événement :

\begin{abstract}
Le 26, le comte de Saint-Aignan, premier gentilhomme de la chambre du roi, chevalier des Ordres de Sa Majesté et gouverneur de Touraine, fut au Parlement, pour l'entérinement de la grâce que Sadite Majesté lui a accordée pour la vigoureuse action, en laquelle, en se défendant contre quatre hommes qui l'avaient attaqué seul, avec pistolets et épées, près de l'une de ses maisons, il en avait tué trois et mis l'autre en fuite ; ayant été accompagné de tant de personnes de qualité qu'il ne s'était point vu d'assemblée si nombreuse en aucune occasion semblable. ${ }^{26}$
\end{abstract}

$\mathrm{La}$ « vigoureuse action » de Saint-Aignan signale son courage et sa force. Elle sera d'ailleurs mentionnée aux côtés de ses faits d'armes au service du roi dans la lettre patente qui le fera duc et pair quelques mois plus tard. En 1664, il y fait allusion en s'attribuant le rôle de Guidon le Sauvage dans la fête des Plaisirs de l'île enchantée : ce chevalier est en effet connu pour avoir triomphé de dix ennemis en un seul combat avant de déflorer dix pucelles en une nuit. René de Bruc de Montplaisir l'évoque lui aussi dans une ballade qu'il lui envoie en lui offrant un mousqueton :

Parmy les bois et la gaye verdure

Où va cherchant souvent mainte aventure

Ainsy que vous tout gentil chevalier

Lorsque seulet vous alliez vous esbattre

Quatre assassins venant vous espier

\footnotetext{
${ }^{24}$ A. Félibien, Relation de la Feste de Versailles du 18 juillet 1668, Paris, Imprimerie royale, 1679, p. 8.

25 Sur la fête de 1674, voir Louis Marin, Le Portrait du roi, Paris, Éditions de Minuit, 1981, « Le roi magicien ou la fête du prince », p. 236-250.

${ }^{26}$ La Gazette (N 77$)$, du 30 juin 1663, p. 614.
} 
Vous avez fait dit on le diable à quatre. ${ }^{27}$

Comme dans la fête de 1664, l'attaque dont Saint-Aignan est victime est ici comparée aux aventures des chevaliers. La violence du duc - le meurtre de trois hommes, pour lequel il doit solliciter la grâce royale - devient l'objet d'un badinage. L'écriture publie la force du duc - quelques vers plus loin, il est comparé à Hercule - et la rend plaisante, notamment par le truchement d'une référence à l'univers romanesque de la chevalerie. La ballade, comme les récits de fête mais à une autre échelle, convertit ainsi la violence en plaisir pour construire la figure d'un pouvoir agréable.

Dans ses Mémoires, le marquis de La Fare raconte lui aussi l'épisode en le rapportant à un univers romanesque. Mais la référence à la fiction, loin de rendre la violence du duc plaisante, est ici utilisée pour la rendre incroyable :

Le duc de Saint-Aignan était le confident des amours du roi ; du reste comparable à Dom Quichotte, car il fit un beau jour assembler le parlement et toute la France pour faire entériner une grâce qu'il avait obtenue pour avoir tué, il y avait longtemps, cinq [sic] hommes lui tout seul; si bien qu'un conseiller de la grand'chambre, à qui on demandait son opinion, ne répondit autre chose, si ce n'est: "Cet acte gigantesque est certes merveilleux. $»^{28}$

L'emploi des adjectifs «gigantesque» et «merveilleux» renvoie bien à l'esthétique de l'aventure. Mais l'assimilation de cette esthétique à un univers romanesque est ici utilisée contre le duc : sa bravoure s'en trouve discréditée. L'aventurier devient un Don Quichotte : un imposteur ou un fou. La mention initiale des « amours du roi » fait en outre écho à l'équivocité de l'aventure telle qu'elle est présentée dans les récits de fêtes. Le discrédit jeté sur l'aventure de Saint-Aignan se double de l'allusion à son rôle dans les aventures érotiques du roi. Les diverses dimensions de l'aventure mobilisées par les récits de fêtes pour construire la figure d'un pouvoir agréable sont ici mises en tension et retournées les unes contre les autres, dans une démarche critique. On peut observer la même opération dans une chanson satirique dirigée contre Saint-Aignan et recueillie par Tallemant des Réaux :

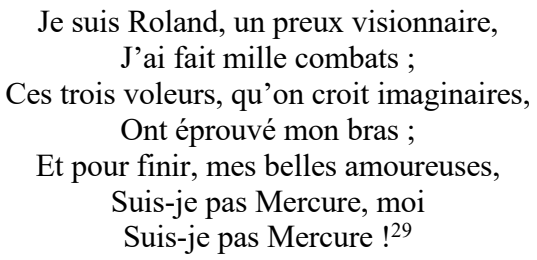

La référence romanesque est mobilisée pour discréditer la vertu guerrière de Saint-Aignan, qualifié de «preux visionnaire », c'est-à-dire de brave imaginaire. Au modèle de l'aventurier valeureux (« Je suis Roland») se substitue alors celui de l'entremetteur («Suis-je pas Mercure ») au service des aventures érotiques du roi. Ces textes dirigés contre Saint-Aignan défont l'alliance de la vertu guerrière et du plaisir. L'usage d'une esthétique romanesque est interprété comme le signe d'une imposture et le plaisir, qu'il soit érotique ou de lecture, est désigné comme incompatible avec la violence guerrière.

L'exploitation des ambivalences de l'aventure et de la tension entre éthique guerrière et esthétique galante n'est pas le propre des textes critiques ou satiriques. En 1670, le Divertissement royal offert par Louis XIV à sa cour à Saint-Germain joue de l'opposition et de l'alliance de la force et des plaisirs ${ }^{30}$. La comédie de Molière qui lui sert de cadre, Les Amants magnifiques, oppose un héros guerrier, Sostrate, et deux princes galants. Tous trois cherchent à obtenir la main de la princesse Ériphile. Sostrate est dépeint comme un aventurier : brave, fort, il cherche la gloire dans les combats militaires. S'il incarne les valeurs éthiques liées à l'aventure, il n'en maitrise pas l'esthétique : il parle peu et fuit les divertissements. Les deux princes au contraire offrent à leur maîtresse des fêtes merveilleuses pour la séduire. Mais cette maîtrise esthétique est dénoncée. D'abord, les princes l'utilisent pour tromper aussi bien que pour plaire. À l'acte IV, l'un des princes utilise une machine de théâtre pour faire croire à une apparition divine. Il espère ainsi tromper la reine et l'encourager à lui donner sa fille. L'un des divertissements merveilleux livrés à l'admiration des spectateurs est donc désigné, à l'intérieur de la fable, comme une illusion

\footnotetext{
${ }^{27}$ Cette ballade est imprimée comme une chose déjà ancienne dans le Mercure galant de novembre 1678 [tome 11], p. 269272.

${ }^{28}$ Charles Auguste, Marquis de La Fare, Mémoires et Réflexions du Marquis de La Fare sur les principaux événements du règne de Louis XIV, [Rotterdam, G. Fristch, 1716], Émile Raunié (éd.), Paris, G. Charpentier, 1884, p. 55-56.

${ }^{29}$ G. Tallemant des Réaux, Le Manuscrit 673, V. Maigne (éd.), Paris, Klincksieck, 1994, p. 367.

${ }^{30}$ Voir L. Naudeix et A. Piéjus, Notice des Amants magnifiques, dans Molière, Euvres complètes, éd. cit., t. II, p. 1677-1687 et M. Roussillon, « Roi danseur, roi guerrier : force et galanterie dans le Divertissement royal », dans L. Naudeix, Molière à la cour : Les Amants magnifiques en 1670, Presses universitaires de Rennes, à paraître.
} 
trompeuse. En outre, la maîtrise esthétique des princes ne leur permet pas de plaire à l'héroïne. Le charme ne fonctionne pas. Pire, la répétition des divertissements au fil de la pièce semble en amoindrir l'effet. La reine les commente ainsi au début de l'acte III : « Les mêmes paroles toujours se présentent à dire. Il faut toujours s'écrier : "Voilà qui est admirable, il ne se peut rien de plus beau, cela passe tout ce qu'on a jamais vu." 11 » Comment mieux dire qu'à force de répétition, il n'y a plus ni surprise, ni merveille ? Au contraire, ce sont la force et la bravoure de Sostrate qui finissent par apparaître comme merveilleuses. À la fin de la pièce, celui-ci sauve la reine en se battant contre un sanglier et obtient ainsi d'épouser Ériphile. Le combat est alors désigné comme une «aventure merveilleuse $^{32} »$. Celle-ci cependant n'a pas sa place sur scène. Le combat n'est même pas raconté. Entre la condamnation des plaisirs trompeurs et l'exclusion de la violence, la pièce construit ainsi dans un jeu de distinctions l'espace d'une force entièrement convertie en plaisir. C'est ce que donne à voir le dernier intermède du Divertissement royal, un ballet représentant les jeux pythiens, dans lequel les danseurs miment exercices de force et combats guerriers à la gloire d'Apollon et de Louis XIV.

Dans les récits de fêtes, l'aventure est définie dans une double relation à la pratique guerrière et à la fiction romanesque. Il s'agit de revendiquer la force et la bravoure du roi et des courtisans qui l'entourent et de faire de cette force l'objet d'un plaisir partagé, non seulement par les spectateurs des divertissements, mais au-delà par tous ceux qui lisent le récit, de manière à susciter l'adhésion la plus large au pouvoir royal. Dans les premières années du règne, les courses et leurs récits se substituent à la gloire militaire encore absente dans le portrait du jeune roi. Après 1667 et la reprise d'une politique de guerre, le roi cesse de participer à des courses. Dans les récits de divertissements, il ne s'agit plus tant d'affirmer la puissance militaire du roi que de revendiquer son caractère agréable, de convertir la violence guerrière en plaisir partagé. Pour opérer la conversion, les récits empruntent à la fiction une esthétique galante, fondée sur le merveilleux et la surprise. Les récits de fêtes, loin d'être de simples documents sur les évènements, produisent leur propre représentation du pouvoir. Les lire à travers la notion d'« aventure » met en lumière leur cohérence esthétique et leur parenté avec d'autres genres modernes et galants, comme la tragi-comédie, le poème héroïque, l'opéra ou le conte de fées. Les ambigüités du plaisir ainsi suscité peuvent être exploitées pour critiquer le pouvoir : l'aventure héroïque du pouvoir est alors assimilée à une fiction ou dégradée en aventures érotiques. La notion d'aventure est ainsi traversée par un débat sur le plaisir et ses usages politiques.

\footnotetext{
${ }^{31}$ Molière, Les Amants magnifiques, III, 1, Euvres complètes, éd. cit., t. II, p. 977.

${ }^{32}$ Ibid., p. 989.
} 